\title{
Multicomponent Assessment of the Geometrical Uncertainty and Consequent Margins in Prostate Cancer Radiotherapy Treatment Using Fiducial Markers
}

\author{
Pablo Castro1, María Roch ${ }^{1}$, Almudena Zapatero², David Büchser 3 , Julia Garayoa4, \\ Cristina Ansón'1, David Hernández¹, Carlos Huerga ${ }^{5}$, Margarita Chevalier6, \\ Saturnino González ${ }^{7}$, Leopoldo Pérez ${ }^{1}$
}

\footnotetext{
${ }^{1}$ Department of Medical Physics, Hospital Universitario la Princesa, Health Research Institute IIS-IP, Madrid, Spain ${ }^{2}$ Department of Radiation Oncology, Hospital Universitario la Princesa, Health Research Institute IIS-IP, Madrid, Spain ${ }^{3}$ Department of Radiation Oncology, Hospital Universitario Cruces, Barakaldo, Spain ${ }^{4}$ Department of Radiological Protection, Hospital Universitario Fundación Jiménez Díaz, Madrid, Spain ${ }^{5}$ Department of Medical Physics and Radiological Protection, Hospital Universitariola Paz, Madrid, Spain ${ }^{6}$ Medical Physics Group, Radiology, Rehabilitation and Physiotherapy Department, Complutense University of Madrid, Madrid, Spain

${ }^{7}$ Department of Radiology, Hospital Universitario la Princesa, Health Research Institute IIS-IP, Madrid, Spain

Email: pablo.castro@salud.madrid.org
}

How to cite this paper: Castro, P., Roch, M., Zapatero, A., Büchser, D., Garayoa, J., Ansón, C., Hernández, D., Huerga, C., Chevalier, M., González, S. and Pérez, L. (2018) Multicomponent Assessment of the Geometrical Uncertainty and Consequent Margins in Prostate Cancer Radiotherapy Treatment Using Fiducial Markers. International Journal of Medical Physics, Clinical Engineering and Radiation Oncology, 7, 503-521. https://doi.org/10.4236/ijmpcero.2018.74043

Received: September 27, 2018 Accepted: November 17, 2018 Published: November 20, 2018

Copyright $\odot 2018$ by authors and Scientific Research Publishing Inc. This work is licensed under the Creative Commons Attribution International License (CC BY 4.0).

http://creativecommons.org/licenses/by/4.0/ (c) (i) Open Access

\begin{abstract}
The aim is to compute all sources of geometrical uncertainty in prostate radiotherapy using fiducial markers and determine the safety treatment margins. Based on the markers position, correlations between prostate rotation/deformation and rectal and bladder fillings as well as changes in prostate volume during the treatment course are analyzed. The study includes 375 pre-treatment CBCT images from 15 prostate cancer patients treated with hypofractionated radiotherapy. The position coordinates of the markers were obtained from each image acquisition. In addition, rectum and bladder were outlined on CBCTs. The intrafractional error was estimated by an additional post-treatment CBCT acquired on alternate days. Tau-Kendall analysis was performed to correlate organ fillings with prostate rotation/deformation. Delineation uncertainty was assessed from contours of 10 patients performed by two radiation oncologists and repeated twice. The CT contouring was assisted by a multiparametric MR approach combining a T2-weighted with diffusion-weighted imaging, and a gradient recalled echo for fiducial marker identification. Uncertainty associated to treatment unit was estimated from phantom measurements. The obtained clinical margins were 4.4, 7.3, $5.1 \mathrm{~mm}$ in the Left-Right, Superior-Inferior, and Anterior-Posterior directions, re-
\end{abstract}


spectively, being the contouring the most important contribution. The mechanical limitations of the beam delivery system and the associated imaging device entailed errors of the same order as prostate motion, rotation or deformation. Weak correlations between variation of the rectal volume and the presence of rotations/deformations were found (correlation coefficient 0.182 , $\mathrm{p}=0.001$ for rotations around lateral axis; correlation coefficients $0.1, \mathrm{p}<$ 0.05 for deformations). The distance between markers decreased with session number, becoming more pronounced from fraction 13 and reaching $1-1.8$ $\mathrm{mm}$ at the end of the treatment. In summary we have determined the optimal treatment margins based on geometrical uncertainty assessment using van Herk formalism. An appropriate preparation of rectum and bladder involves minimizing the effect of prostate rotations/deformations. The prostate tends to decrease in size during the treatment which could influence treatment re-planning strategies.

\section{Keywords}

Treatment Margin, Fiducial Marker, Prostate, Geometrical Uncertainty

\section{Introduction}

Radiotherapy (RT) is currently a routine modality in the treatment of prostate cancer. Randomized trials evidence the benefit of higher radiation doses in clinical outcomes improving local control and reducing the risk of biochemical failure for prostate cancer patients. In contrast, this increment in the delivered dose may increase the incidence of urinary and rectal complications. However, the advances experienced in recent years have made this dose escalation technically possible without an increase in toxicity [1] [2]. In particular, volumetric arc modulation (VMAT) provides high dose conformation in the treatment volume while allowing high dose gradients and preservation of adjacent healthy tissue. Because of the special features of these distributions an accurate location of the target volume is required. Throughout the radiotherapy process the patient's geometry is subject to numerous sources of uncertainty that make this objective difficult to achieve. In order to ensure the effectiveness of treatment, it is necessary to add a margin to the initial volume CTV to take into account these uncertainties. Different methods have been described to correlate the geometrical uncertainty and the treatment margin that are usually synthesized in margin recipes [3] [4].

One of the most important contributions to geometrical inaccuracy is the reproducibility of patient positioning between sessions. The use of image-guided radiotherapy techniques (IGRT) allows relating the position of the treatment volume to the radiation beam and, therefore, to reduce the uncertainties associated to the positioning in the treatment unit. In fact, the PTV margins are closely related to the IGRT strategy used. More specifically, the improvement in treatment accuracy in prostate patients has been demonstrated by the implanta- 
tion of intraprostatic fiducial markers [5]. The markers can be used as a surrogate for the prostate itself and their position is easily identified in the image. Ideally, positioning errors can be removed by using fiducial markers. Nevertheless, in clinical practice there are residual errors that cannot be corrected. These residual errors must be known and quantified. Examples of these errors may be the detection limit of the image device, imprecise movement of the repositioning system, differences between treatment and image isocenters, and limitations associated to image registration. Other potential sources of uncertainty associated to the treatment machine are gantry and collimator rotation inaccuracy. In addition, the gland may undergo deformations and movements during treatment which are also part of the geometrical uncertainty. Finally, the uncertainty in the contour delineation depends on the interobserver variability for the different image modalities employed.

The objective of the study was to analyze the components of the geometrical uncertainty that participate during the entire radiotherapy process. Based on the markers position the correlation between prostate rotation/deformation and changes in rectal and bladder fillings was also analyzed. Changes in prostate volume during the treatment course were included in the study. As a last aim the safety margins of the treatment volume that ensure the efficacy of the RT treatment in patients with prostate cancer were also estimated.

\section{Material and Methods}

In order to estimate the uncertainties associated to the different components, we perform a statistical analysis of phantom and patient data. Phantom data were employed to quantify errors associated to imaging and treatment delivery systems. Patient information was extracted from positioning imaging before and after the treatment session.

\subsection{Phantoms}

Two different phantoms were used to assess the uncertainty related to the treatment machine. The QUASAR Penta-Guide phantom (ModusMedical Devices, Canada) is a specific phantom for quality control of IGRT techniques. It contains three hollow spheres fixed at known positions. The spheres produce high-contrast images due to the large density difference between the air inside them and the phantom material. Crosshairs on each phantom face allow aligning its geometric center with the room lasers isocentre. The Alderson RANDO phantom (Radiology Support Devices, USA) is an anthropomorphic phantom transected horizontally into $2.5 \mathrm{~cm}$ thick slices. The slices consist of soft-tissue-equivalent material on which bony structures and air cavities are embedded in order to mimic the human anatomy in terms of shape and density.

\subsection{Patients}

\subsubsection{Simulation}

Before the CT simulation, prostate cancer patients underwent the implantation 
of three gold seeds by ultrasound-guided insertion; one at the apex, S1, and two at each side of the prostate base, S2 left and S3 right. Two weeks later, CT images were acquired for treatment planning using an Aquilion LB scanner (Canon Medical Systems, Japan). The study was performed in supine position using an indexed leg-immobilization device with a slice thickness of $2 \mathrm{~mm}$. The patient was instructed to have comfortably filled bladder and empty rectum for both simulation and treatment.

\subsubsection{Target Definition and Treatment Planning}

The software used to perform these tasks was the treatment planning system Eclipse v11.0 (Varian Medical Systems, USA). For the prostate delineation, the CT study was used together with an MR image set. Patients were equally immobilized in CT and MR, and they were instructed to follow the same bladder and rectum regimen. Three MR sequences were acquired using a GE 1.5T Signa Infinity scanner MR (General Electric Healthcare, UK); a T2-weighted image and a diffusion-weighted image (DW) for prostate delineation and a gradient recalled echo (GRE) for fiducial markers identification. To minimize variation in the anatomy of the patient, the MR study was acquired within 3 days immediately after the acquisition of the CT. Fiducial markers were identified by a hyperintense signal in CT and by signal voids in GRE MR [6] [7]. Then both studies were registered based on a match point registration. Contouring was performed on the CT/MR image fusion. Once the prostate was outlined, the treatment was planned using VMAT with two 360-degree arcs of 6MV-energy, $600 \mathrm{UM} / \mathrm{min}$ maximum rate and collimator angles at $30^{\circ}$ and $330^{\circ}$. A hypofractionated treatment of 67.5 Gy was scheduled in 25 sessions, with a simultaneous boost of 69 Gy.

\subsubsection{IGRT Equipment and Positioning Protocol}

The treatment delivery unit was a Varian Clinac 2300 iX accelerator equipped with the On Board Imaging, OBI (Varian Medical Systems, USA). The daily procedure for patient positioning consists of acquiring a 3D kilovoltage image using the OBI system. This allows performing the Cone Beam CT (CBCT) technique. In all cases, phantom or patient, the pelvis protocol was performed: a 360-degree СВCT with half-bowtie filter and detector offset, followed by image reconstruction on a $512 \times 512$ matrix, and $2 \mathrm{~mm}$ slice thickness. Next, the CBCT was registered with the reference CT using the OBI system software.

In theory, the daily frequency of the protocol eliminates interfraction errors. The system provides the translational mismatches to correct positioning errors after obtaining the best matching between markers in the acquired СВСТ and reference CT. Translational correction was achieved by moving the treatment table on the three axes of movement: Left-Right (LR), Superior-Inferior (SI), and Anterior-Posterior (AP). Before correction, the CBCT image was used to verify that the rectum and bladder preparation was consistent with that from the simulation. In case the patient did not comply with the requirements of bladder 
and rectal filling, the treatment was delayed until an appropriate preparation. The isocenter and markers position coordinates were extracted from all the CBCT and CT studies. We selected 15 consecutive patients with localized prostate cancer treated with the hypofractionated treatment scheme mentioned above as the only inclusion criterion. The positioning errors obtained from 375 $\mathrm{CBCT}$ were employed in the analysis. The rectum and bladder were contoured in 173 CBCTs aiming to determine the impact of the filling of adjacent organs on the prostate rotations and deformations.

For the 15 patients and on alternate days, an additional post-treatment CBCT, followed by the corresponding registration of fiducial markers, was acquired to study the intrafractional prostate motion. Again, the markers position coordinates were extracted from each post-treatment $\mathrm{CBCT}$ and compared with the coordinates computed in the pre-treatment СBCT. Specifically, the intrafraction motion errors obtained from $170 \mathrm{CBCTs}$ were analyzed.

\subsection{Components of Geometrical Uncertainty}

\subsubsection{Isocenter Coincidence}

To perform an accurate IGRT the isocenter of the $\mathrm{kV}$ image system has to be as close as possible to the isocenter of MV radiation. The alignment of both isocenters is checked monthly with the Quasar phantom following a reported procedure [8] [9]. A CT scan of the phantom is acquired and a 4-field box treatment is planned. The isocenter is selected in the centroid of the central air sphere. In the treatment unit, the geometric center of the phantom is aligned with the isocenter indicated by the lasers. $6 \mathrm{MV}$ X-rays portal images are acquired at each gantry angle $\left(0^{\circ}, 90^{\circ}, 180^{\circ}\right.$, and $\left.270^{\circ}\right)$. The images are acquired at $90^{\circ}$ and $270^{\circ}$ collimator rotation in order to avoid potential asymmetries in the jaws position. The centroid of both the central sphere and the radiation field is calculated on the image (centroids of each pair of collimator projections are averaged). This difference provides the discrepancy between the isocenter indicated by the lasers and the MV isocenter (vector $\overrightarrow{\Delta_{1}}$ ). Then a CBCT is acquired and registered with the planning CT. The obtained correction shifts show the discrepancy between the isocenter indicated by the lasers and the $\mathrm{kV}$ reconstruction isocenter (vector $\left.\overrightarrow{\Delta_{2}}\right)$. Finally, the vector resulting from the difference $\left(\overrightarrow{\Delta_{1}}-\overrightarrow{\Delta_{2}}\right)$ shows the discrepancy between the $\mathrm{kV}$ isocenter and the MV isocenter.

\subsubsection{Isocentric Rotation}

Prostate cancer treatment in our center is composed of two complete arcs at $30^{\circ}$ and $330^{\circ}$ collimator angles, without table rotation. The component associated with the geometrical inaccuracy of the treatment unit during irradiation is estimated from previously acquired portal images at the 4 principal gantry angles and each at collimator angles $90^{\circ}$ and $270^{\circ}$. The centroid of the radiation field is determined in the images, and the centroids of each pair of projections are averaged. The average of all centroids is considered the center of rotation. The deviation from the center of rotation is determined for each axis, since it is easy to 
separate the deviation by component, LR, SI, and AP, for the chosen angles. The maximum deviation found in the four fields was taken as the estimator of the uncertainty. The table rotation can be neglected since only coplanar beams were used.

\subsubsection{Residual Error of the Imaging System. Uncertainty in the Table Movement}

The imaging system has its own imperfections that impose a limit on detectability and represent a specific uncertainty. We include in this uncertainty the following components: imaging system pixel size, deformation in the image reconstruction, and finally the uncertainty in the table shift. The latest has mainly a random nature which results from rounding the calculated table shifts into integer millimeters and the imprecise execution of these table movements. To estimate it, we assigned a uniform probability distribution in which the variation limits are associated to the table movement resolution. This distribution gives a constant value inside the interval and a null value outside.

The other uncertainty components were determined by using the anthropomorphic phantom. A CT scan of the pelvic region was acquired with radiopaque markers placed on the surface. The planning isocenter was placed at the markers intersection point. Sub-millimeter precision rulers placed on the phantom surface allow us translations with an estimated resolution of $0.25 \mathrm{~mm}$ (based on visual discrimination threshold), better than that given by the treatment unit (1 $\mathrm{mm})$. A CBCT was acquired after phantom alignment in the treatment unit. The translational shifts were obtained by performing a rigid registration with the reference CT. Next, using the rulers, known displacements (in $\mathrm{cm}$ ) were made in each direction and sense, first separately, $\pm 1.0, \pm 0.5, \pm 0.2$, and then combining them $( \pm 0.5, \pm 0.5,0.0),( \pm 0.5,0.0, \pm 0.5)$ and $(0.0, \pm 0.5, \pm 0.5)$. The CBCT in each position was repeated 3 times. The registration was done automatically matching the pelvic region. Because there were no deformations in the phantom, the uncertainty in the registration was very close to zero. The uncertainty associated to the markers registration was intrinsically included in the set-up residual error. The errors were determined from the difference between the shift measured with the ruler and the correction shift provided by the Varian registration software. The obtained statistical results were quadratically combined with the table resolution uncertainty to obtain the residual error of the system.

\subsubsection{Set-Up Residual Error}

In addition to being representative of the prostate position, fiducial markers have the advantage of being easily located and reducing the interobserver variability in the alignment of the images [10] [11]. In each treatment session, the prostate may undergo deformations that can lead to relative displacements between markers different from those of the CT simulation. In our clinical practice, a rigid registration based exclusively on markers is performed. The problem, therefore, is reduced to a rigid solid with three degrees of freedom, transla- 
tions in three axis (rotations are not considered quantitatively). Ideally, if there is no prostate deformation and no seeds migrations, and the registration is accurate then the relative position of the markers with respect to both isocenters should coincide. In order to quantify this potential error, it is calculated the difference between the markers center of mass, $\mathrm{CM}_{\text {markers }}$, in the $\mathrm{CBCT}$ relative to the treatment isocenter and $\mathrm{CM}_{\text {markers }}$ in the $\mathrm{CT}$ relative to the planning isocenter. $\mathrm{CM}_{\text {markers }}$ is computed from the markers position coordinates.

\subsubsection{Prostate Rotation}

The prostate can rotate causing a possible underdose in the treatment volume. Although the rotations are partially compensated by the table shifts, there is a residual rotation difficult to correct without using a six degree couch. The effect of rotation on target coverage will depend on the magnitude of rotation, the distance from rotation center to target center of mass, $\mathrm{CM}_{\text {target, }}$ and extension of their contours in the three spatial directions. In the present study, the magnitude of the rotation with respect to the markers center of mass, $\mathrm{CM}_{\text {markers }}$, was determined. From the markers coordinates relative to $\mathrm{CM}_{\text {markers }}$ we can deduce the angle formed by axis of rotation. For example, for the lateral axis, the rotation angle of the marker S3 is given by $\operatorname{arctang}(y / z)$, where $\mathrm{y}$ and $\mathrm{z}$ are the spatial coordinates of S3 in SI and AP directions, relative to the $\mathrm{CM}_{\text {markers }}$. The difference between the angle measured in the $\mathrm{CT}$ and that measured in the $\mathrm{CBCT}$ for the corresponding session determines the residual rotation related to the marker. The residual angle is taken as the average of the angles obtained by each marker. Since it is a marker-based registration, the effect of the rotation depends on the distance between the $\mathrm{CM}_{\text {markers }}$ and the $\mathrm{CM}_{\text {target }}$. The translation error due to uncorrected rotations in the $\mathrm{CM}_{\text {markers }}$ can be estimated according to [12]:

$$
\text { error }=\mathrm{D} \cdot \sqrt{2 \cdot(1-\cos \theta)}
$$

where $\mathrm{D}$ is the distance between $\mathrm{CM}_{\text {markers }}$ and $\mathrm{CM}_{\text {target }}$ and $\theta$ is the rotation angle.

Figure 1 shows the magnitudes involved in the calculation of rotations and associated errors.

Secondly, we analyzed whether the prostate rotations at the different sessions are correlated with changes experienced in the filling of bladder and rectum. The volumes of the rectum and bladder were inferred from the contours delineated in the СBCT. The volume differences from planning $\mathrm{CT}$ were calculated resulting $\Delta \mathrm{V}_{\text {rectum }}$ and $\Delta \mathrm{V}_{\text {bladder }}$. Possible effect on prostate rotation due to changes of rectal and bladder volumes $\Delta \mathrm{V}_{\text {rectum }}$ and $\Delta \mathrm{V}_{\text {bladder }}$ were evaluated by means of the Tau-Kendall correlation coefficients.

\subsubsection{Prostate Deformation}

The prostate markers have been considered as representatives of its position. However, the gland can change in size and shape during the treatment. We have estimated the residual errors associated to prostate deformations by assuming that the markers and the surface of the prostate move together [13]. The distance 


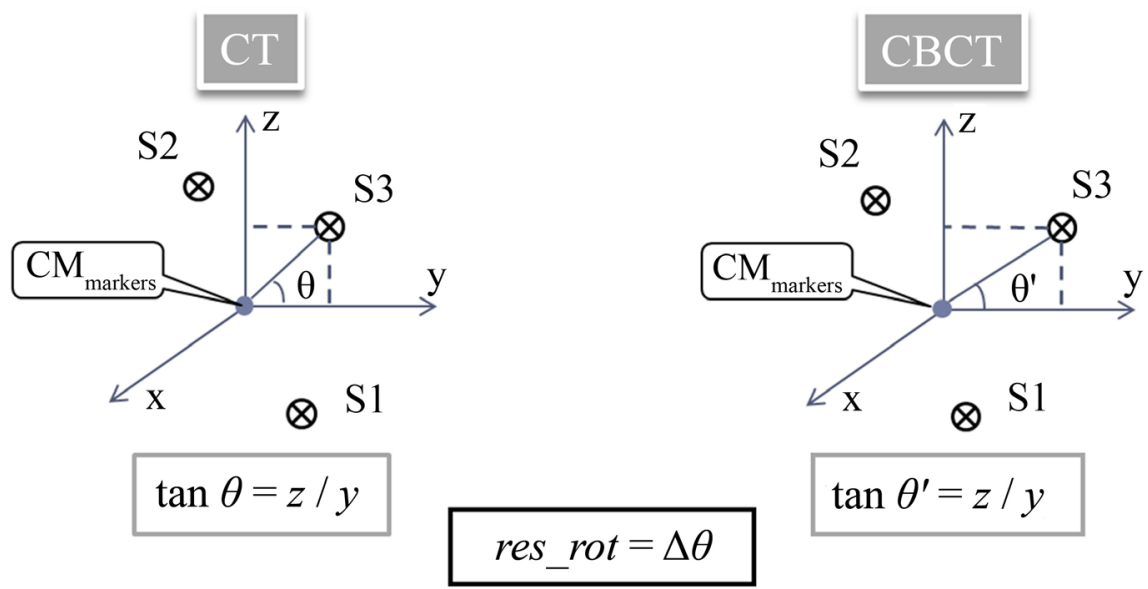

(a)

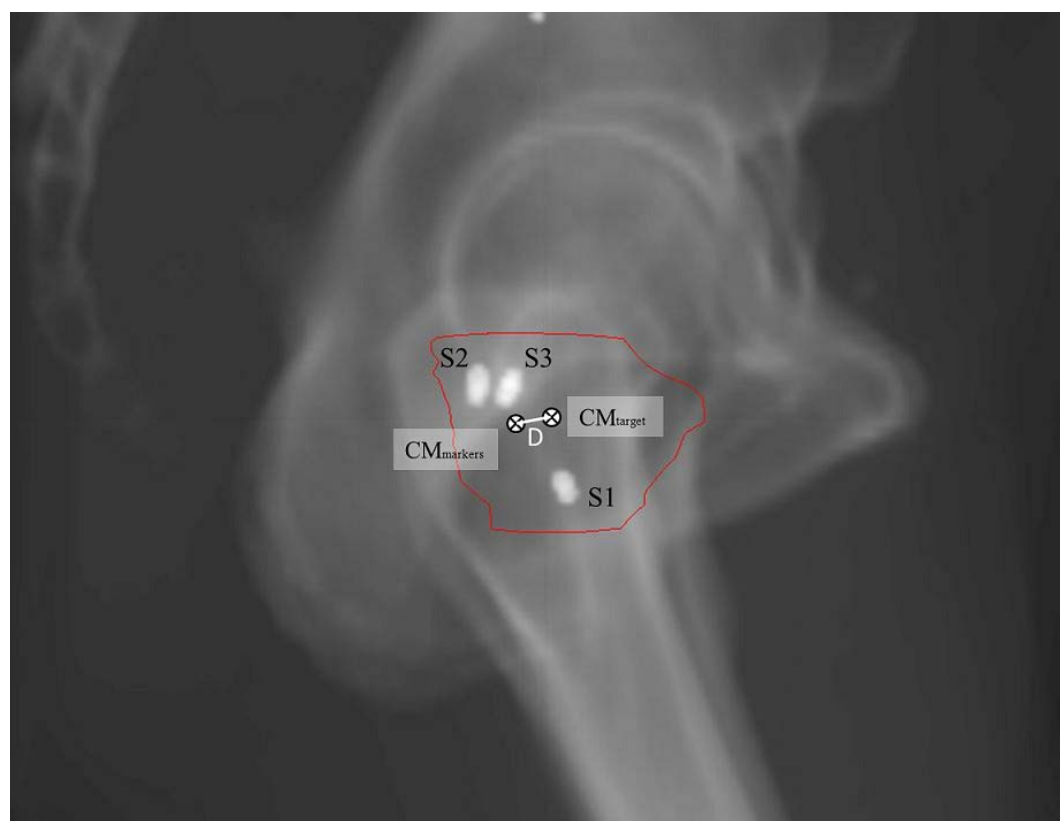

(b)

Figure 1. (a) Diagram showing the fiducial markers (S1, S2, and S3) and the center of mass. Definition and calculation of the rotation angle $\theta$ around the $\mathrm{YZ}$ axis for S3 both in the CT study and in the CBCT, and the associated residual rotation are included; (b) Lateral beam's eye view where fiducial markers and prostate contour can be visualized. The distance between the center of mass for the fiducial markers, $\mathrm{CM}_{\text {markers }}$, and the center of mass for the prostate contour, $\mathrm{CM}_{\text {target }}$, is represented by the white line $\mathrm{D}$.

of each marker to the $\mathrm{CM}_{\text {markers }}$ was calculated from the position coordinates of the markers. The difference between these distances in CT and CBCT was determined for each treatment session. As an estimator of the residual error we took the maximum value obtained by axis, neglecting the negative values that implied a reduction of distances.

Additionally, the separation distance was calculated two by two; $\mathrm{D}_{12}$ distance between $S 1$ and $S 2, D_{13}$ distance between $S 1$ and $S 3, D_{23}$ distance between $S 2$ and S3. The difference between the CT and CBCT distances, $\Delta \mathrm{D}_{12}, \Delta \mathrm{D}_{13}$, and $\Delta \mathrm{D}_{23}$, 
was calculated for each patient and averaged per treatment session to analyze its temporal variation.

From markers coordinates and rectum and bladder contours we analyzed whether organ fillings can cause prostate deformation. The interrelationship between distance variations, $\Delta \mathrm{D}_{12}, \Delta \mathrm{D}_{13}$, and $\Delta \mathrm{D}_{23}$, and $\Delta \mathrm{V}_{\text {rectum }}$ and $\Delta \mathrm{V}_{\text {bladder }}$ was evaluated by Tau-Kendall correlation test for non-parametric distributions.

\subsubsection{Intrafraction Motion Error}

The prostate motion during the irradiation entails an additional uncertainty to be considered in the PTV margin. In our study, systematic and random errors were determined from the displacement of the prostate between initial and final CBCT. However, the fact of considering the range of movement makes us overestimate this component since the prostate does not stay all the time in that position. To have a more realistic estimate we assigned a rectangular distribution to the motion range. Therefore, the probability that the prostate was at a position within the motion range was the same as for the rest of the positions. The associated uncertainty was the value of the motion range divided by the square root of 3 .

\subsubsection{Contouring}

The image resolution, the noise of the observer, the organs motion during the acquisition, and the errors in the image registration when different modalities are used are the main contributions to the uncertainty in the treatment volume delineation. This results in inter-observer or even intra-observer variations. To aid in the task and minimize variations, the contouring was performed on the fusion of the CT image with the RM T2 and RM DW images. Previously, CT and RM GRE images were registered based on a match point registration. Two experienced radiation oncologists $(\mathrm{AZ}, \mathrm{DB})$ with the assistance of an expert genitourinary radiologist $(\mathrm{SG})$ outlined the prostate contours of 10 patients. For this purpose, the CTV definition followed the recently published ESTRO ACROP consensus guideline [14]. The CTV delineation included the prostate and proximal seminal vesicles until the point where the seminal vesicles split without taking into account the $3 \mathrm{~mm}$ expand for extracapsular extension. In general, the intraobserver variability is significantly lower than the interobserver variability [15], so each observer repeated twice the prostate contouring in a time interval superior to 1 week. The contours coincidence for a particular scan was quantified from the measured volumes and the ratio of the encompassing volume and common volume were determined [16]. Next, the perpendicular maximum distance between contours for the same patient was obtained in different regions. From these data, we deduced the values of systematic errors according to the literature [16] [17].

\subsection{Margin Calculations}

There are several formalisms that relate the geometrical uncertainty and the 
treatment margin [3] [4]. One of the most used is based on coverage criteria include dose-probability histograms [3] (named van Herk formalism in this work). This methodology (followed in the present study) distinguishes between systematic errors, $\Sigma$ (producing displacements of the dose distribution with respect to the CTV) and random errors, $\sigma$ (blurring the dose). The margin recipe proposed so that $90 \%$ of patients have a minimum dose in the CTV of $95 \%$ is $M=2.5 \Sigma+$ $0.7 \sigma$, where $\Sigma$ is the quadratic sum of the systematic errors and $\sigma$ is the quadratic sum of the random errors.

\section{Results}

\subsection{Isocenter Coincidence}

The discrepancy between the imaging isocenter and treatment isocenter becomes a systematic error that cannot be corrected. The error is directly transmitted to the patient positioning, and therefore this component is linearly added to the systematic uncertainty. We characterize this component from the results obtained in the periodic quality controls performed on the machine during one year. Average values and the associated standard deviation are shown in Table 1.

\subsection{Isocentric Rotation}

The maximum deviation found from the center of rotation in the radiation fields was taken as the uncertainty estimator. This component was partially included in the previous one, which implies that the associated uncertainty will most likely be overestimated. However, this overestimation will probably not have any relevance if we take into account the other components. The procedure included the isocentrity of gantry and collimator and was easily separated by components. The mean values obtained from 1-year quality assurance were employed in margin calculation as systematic error (Table 1).

\subsection{Residual Error of the Imaging System. Uncertainty in the Table Movement}

The obtained results showed a lack of tendency in residual errors with the magnitude of the shifts. Therefore, the standard deviation obtained was used as statistical estimator of the uncertainty.

For the three axes the resolution in the table movement indicator was $1 \mathrm{~mm}$. Thus, the maximum variation limits were given by $-0.5 \mathrm{~mm}$ and $+0.5 \mathrm{~mm}$, and then the standard uncertainty was given by 0.5 divided by the square root of 3 , if a rectangular distribution was assumed. Residual error and table movement uncertainty were added by quadratic combination. As can be seen in Table 1, results were similar in the three axes.

\subsection{Set-Up Residual Error}

The results for this error including all sessions and patients are shown in Figure

2. The computed systematic and random errors are presented in Table 1 . The 
Table 1. Systematic and random geometrical uncertainty associated to the different components and directions (Left-Right (LR), Superior-Inferior (SI), and Anterior-Posterior (AP)), and required margins using the van Herk formalism.

\begin{tabular}{|c|c|c|c|c|c|c|c|c|c|}
\hline \multirow{2}{*}{ Components } & \multicolumn{3}{|c|}{ Systematic error $\Sigma(\mathrm{mm})$} & \multicolumn{3}{|c|}{ Random error $\sigma(\mathrm{mm})$} & \multicolumn{3}{|c|}{ van Herk margin $(\mathrm{mm})$} \\
\hline & LR & SI & $\mathrm{AP}$ & LR & SI & $\mathrm{AP}$ & LR & SI & AP \\
\hline $\begin{array}{c}\text { Isocenter } \\
\text { coincidence }^{\mathrm{a}}\end{array}$ & 0.51 & 0.12 & 0.62 & & & & 1.6 & 1.0 & 1.6 \\
\hline Isocentric rotation & 0.10 & 0.89 & 0.18 & & & & 0.3 & 2.2 & 0.4 \\
\hline $\begin{array}{l}\text { Residual error } \\
\text { imaging system }\end{array}$ & 0.39 & 0.50 & 0.49 & 0.29 & 0.29 & 0.29 & 1.2 & 1.5 & 1.4 \\
\hline $\begin{array}{l}\text { Set-up residual } \\
\text { error }\end{array}$ & 0.31 & 0.81 & 0.44 & 0.74 & 1.03 & 1.11 & 1.3 & 2.7 & 1.9 \\
\hline Prostate rotation & 0.22 & 0.49 & 0.49 & 0.20 & 0.39 & 0.41 & 0.7 & 1.5 & 1.5 \\
\hline $\begin{array}{c}\text { Prostate } \\
\text { deformation }\end{array}$ & 0.19 & 0.23 & 0.24 & 0.16 & 0.56 & 0.26 & 0.6 & 1.0 & 0.8 \\
\hline $\begin{array}{l}\text { Intrafraction } \\
\text { motion }\end{array}$ & 0.39 & 0.79 & 0.65 & 0.77 & 1.34 & 1.22 & 1.5 & 2.9 & 2.5 \\
\hline \multirow[t]{3}{*}{ Contouring } & 1.0 & 1.4 & 0.9 & & & & 2.5 & 3.5 & 2.3 \\
\hline & \multicolumn{3}{|c|}{ Quadratic sum } & \multicolumn{3}{|c|}{ Quadratic sum } & \multicolumn{3}{|c|}{ Total margin } \\
\hline & 1.32 & 2.14 & 1.54 & 1.13 & 1.85 & 1.75 & 4.4 & 7.3 & 5.1 \\
\hline
\end{tabular}

a. The margin calculated for this component includes the mean value $(0.29,0.68,0.01)$ that is linearly added.

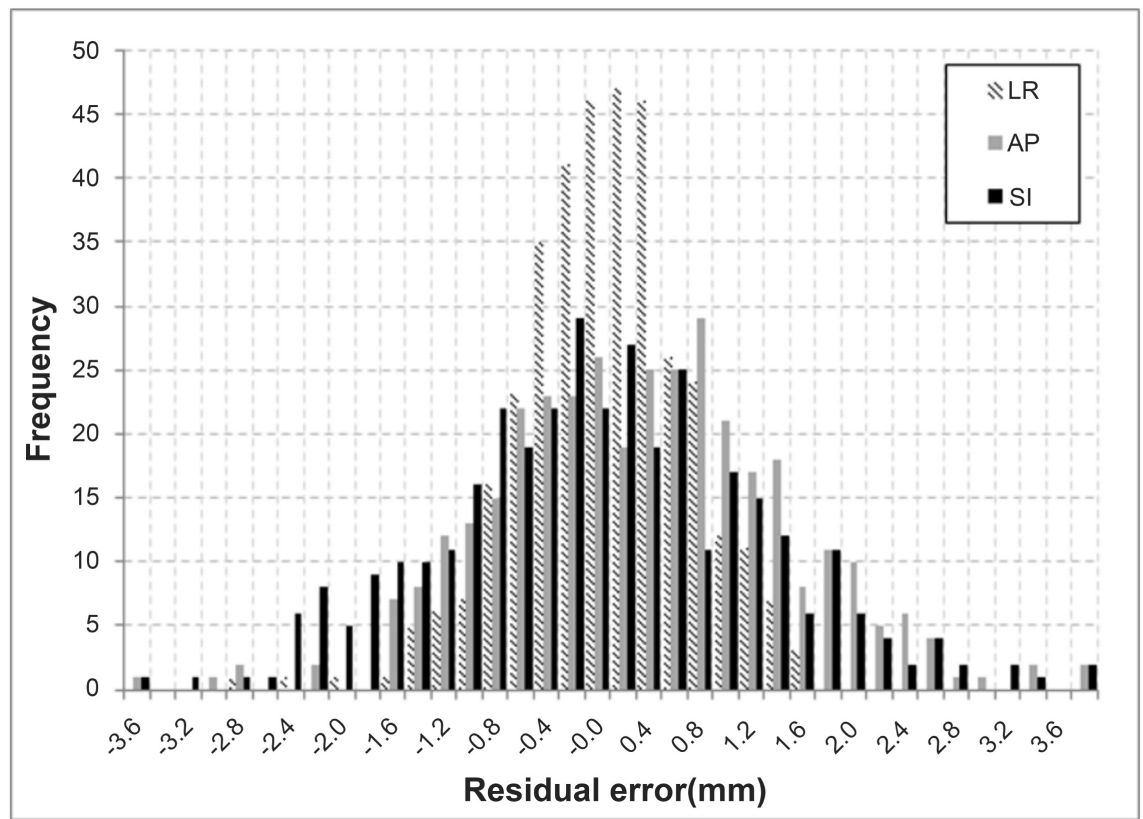

Figure 2. Histogram of the set-up residual error based on the difference between the markers center of mass in the CBCT relative to the treatment isocenter and the markers center of mass in the CT relative to the planning isocenter. Data were obtained from 375 CBCT performed on 15 patients.

higher errors detected in the longitudinal and vertical directions could be explained by the prostate rotations on the lateral axis, which were not completely 
compensated by the translational shifts.

\subsection{Prostate Rotation}

Statistical values, mean value, $\Sigma$, and $\sigma$, obtained for the prostate rotation angle were $1.5^{\circ}, 6.2^{\circ}$, and $3.5^{\circ}$, respectively, for rotation around the lateral axis, $0.5^{\circ}$, $1.8^{\circ}$, and $1.7^{\circ}$, respectively, for XY rotation around longitudinal axis, and $0.3^{\circ}$, $2.5^{\circ}$, and $1.1^{\circ}$, respectively, for $\mathrm{XZ}$ rotation around vertical axis. Both systematic and random components detected in lateral axis were the largest of the three axes, agreeing with other reported data in the literature [12] [18] [19] [20].

The translational error is influenced by rotations around two axes. For example, the lateral component of the residual error is affected by rotations around longitudinal and vertical axes. Therefore, the global translational error was calculated by the quadratic sum of the errors around two corresponding axes. Uncertainties caused by uncorrected rotations are shown in Table 1. SI and AP components presented the largest errors, which correlated with the highest rotation angles found around the lateral axis.

The Tau-Kendall statistical analysis showed a correlation between the rotation around lateral axis and rectal filling (correlation coefficient 0.182; $p=0.001$ ). No significant correlation was found with the other axes of rotation, or between bladder filling and rotation around any axis.

\subsection{Prostate Deformation}

We found that errors due to prostate deformations are equal to or smaller than those due to other components (Table 1). The contribution to this component is mainly provided in the first treatment sessions, since markers distances tend to be reduced over the course of treatment for most patients. The time trend can be observed more clearly in Figure 3, where the average separation distance between fiducials is represented versus the session number. The figure shows that the inter marker distances decreased during the treatment course. This fact can be associated with a potential reduction in prostate volume. Although changes vary unpredictably among patients, around session 13 the average distances tended to decrease, with $\mathrm{D}_{23}$ showing a smaller reduction at the end of the treatment (about $1 \mathrm{~mm}$ ). The principal component of $\mathrm{D}_{23}$ was the lateral component, since both markers were placed approximately at the basis and at the same longitudinal and vertical coordinates. $\mathrm{D}_{12}$ and $\mathrm{D}_{13}$ decreased to around 1.7 $\mathrm{mm}$ in the last session, which means a reduction in the longitudinal axis.

The Tau-Kendall analysis showed a correlation between the deformation related to $\mathrm{D}_{12}$ and $\mathrm{D}_{13}$ distances and rectal filling (correlation coefficient $0.139, \mathrm{p}=$ 0.007 for $\mathrm{D}_{12}$, and correlation coefficient $0.109, \mathrm{p}=0.037$ for $\mathrm{D}_{13}$ ). No significant correlation was found between bladder filling and deformation in any axis.

\subsection{Intrafraction Motion Error}

The intrafraction error was determined from the displacement of the prostate 


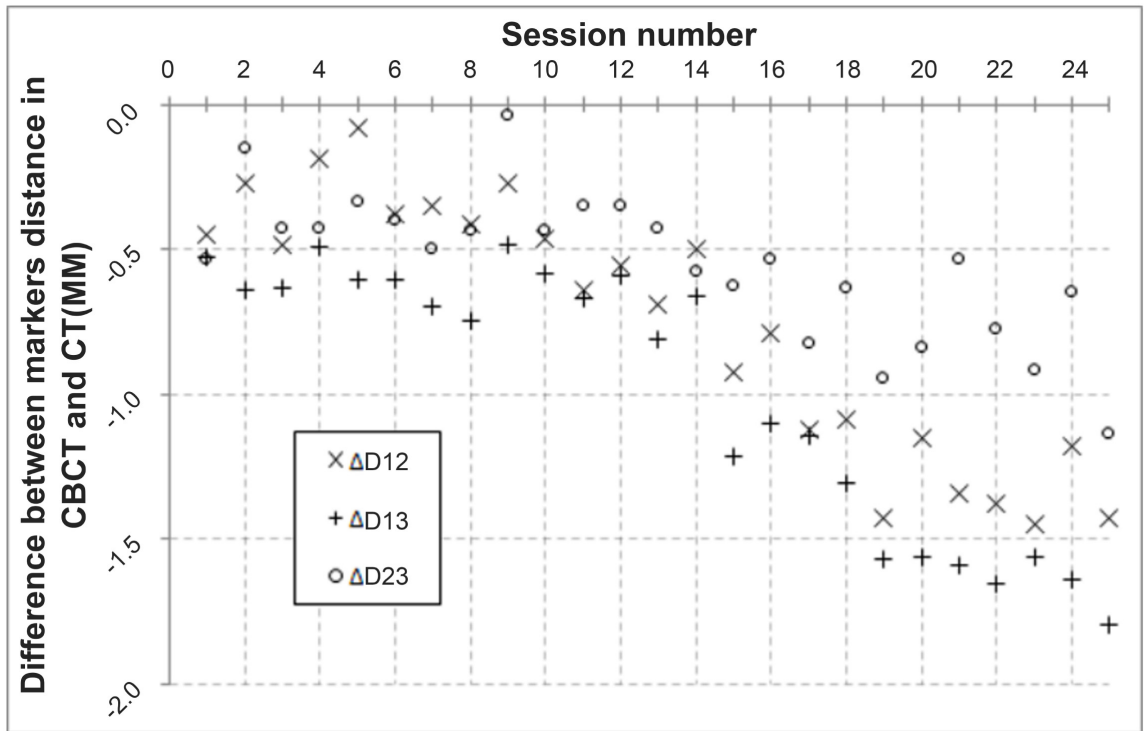

Figure 3. Variation of the separation distance between markers in CBCT and planning $\mathrm{CT}, \Delta \mathrm{D}_{12}, \Delta \mathrm{D}_{13}$, and $\Delta \mathrm{D}_{23}$, and the radiotherapy fraction. $\mathrm{D}_{12}$ is the distance between fiducial S1 and S2, $D_{13}$ the distance between $S 1$ and S3, $D_{23}$ the distance between S2 and S3. S1 is placed at the apex, S2 on the left of prostate base, and S3 on the right of prostate base.

between initial and final CBCT and assuming a rectangular distribution within that range. Figure 4 shows the motion errors obtained from the 15 patients. It can be noticed that the movement of the prostate occurs mainly in the longitudinal and vertical axes which implies that systematic and random errors become larger (Table 1).

\subsection{Contouring}

The mean ratio between the encompassing and common volume was 1.34 when we compared target delineation between observers. If we consider the two repeated contouring by an individual observer, the mean ratio was reduced to 1.17 for both observers, indicating that the intraobserver variation was lower than the interobserver variation. The longitudinal direction presented the largest perpendicular distance between contours. At the base of the seminal vesicles variations close to $1 \mathrm{~cm}$ were found in some cases. The computed systematic error for the interobserver variation was $1.0,1.4$, and $0.9 \mathrm{~mm}$ in the LR, SI, and AP axis, respectively.

\section{Discussion}

The present study aimed to analyze all the components that can contribute to the geometric uncertainty associated the radiotherapy process. Our results show that the contribution due to mechanical limitations of the treatment unit is of the same magnitude of the one associated with the patient, if the contouring stage is not taken into account. Indeed, the contouring is the largest contribution to the geometric uncertainty; therefore a greater effort must be made through 


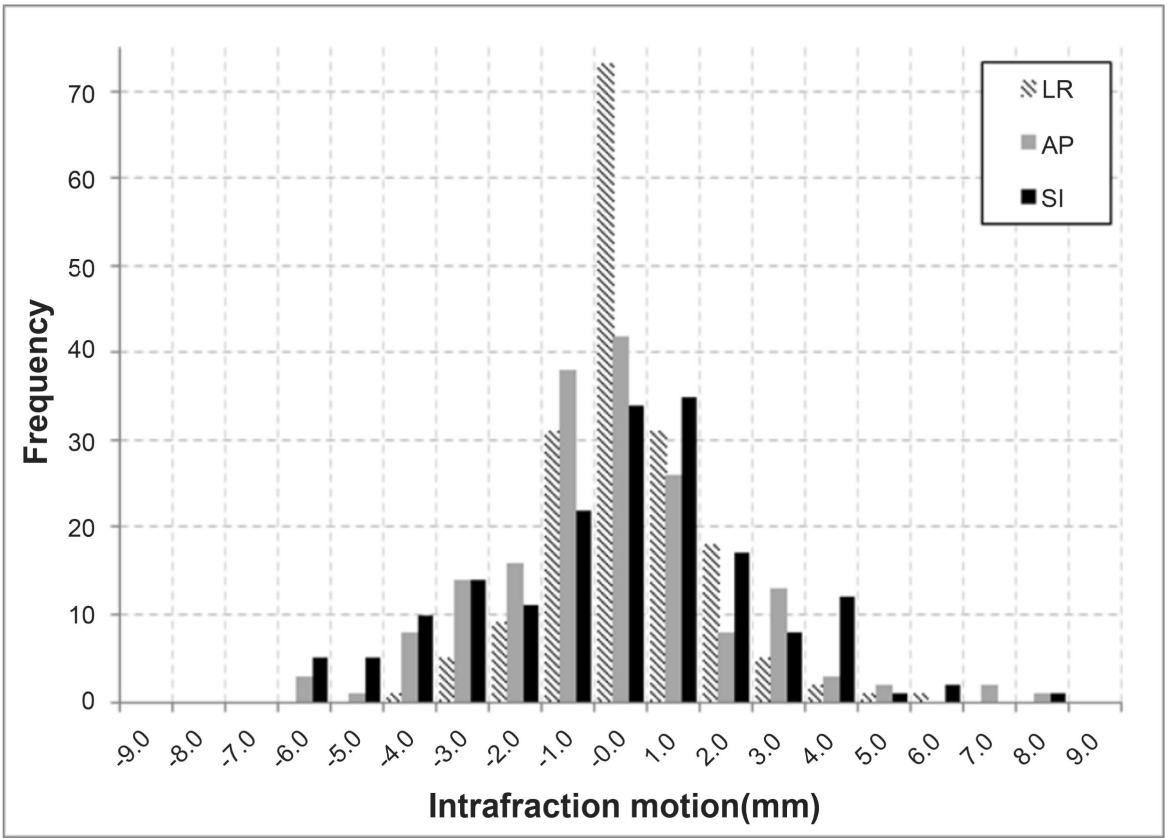

Figure 4. Histogram of the intrafraction motion error determined from the motion range of the prostate between initial and final CBCT. Data were obtained from 340 CBCT (170 pre-treatment and 170 post-treatment) performed on 15 patients.

protocols or guidelines to reduce the interobserver variability. In this sense, it seems that MR provides a greater accuracy in the contour of the prostate [21], although its use also presents limitations. For example, the acquisition time is much longer than that of the CT and there may be differences due to patient movement between sequences and even motion artefacts can be generated. Also the non-exact coincidence in the patient's organ filling complicates the registration between CT and RM. The deformable registration can be a helpful tool for $\mathrm{CT}$ and RM matching. However, it is necessary to consider the additional uncertainty that this type of registration entails, especially taking into account the soft tissue surrounding the prostate and the low contrast between the structures in the CT image. The solution adopted in our center was to perform marker-based registration through a gradient-echo MR image [6] [7], which assists the detectability of the markers and reduces the uncertainty in the registration. Thus, following the ESTRO ACROP guideline on CT/MR based delineation [13], our results show lower systematic errors than other authors [16], which can be related to multimodality imaging but also to the image acquisition parameters (slice thickness of $2 \mathrm{~mm}$ in both CT and MR). The maximum discrepancy between contours was mainly located in the longitudinal direction, at the base of the seminal vesicles and the apex, agreeing to other authors [16] [17].

In the same way, the set-up residual error implies an uncertainty no greater than that of the other components. In general, it can be observed that the largest uncertainties occur in the longitudinal axis, which is probably related to the lowest resolution of $\mathrm{CT}$ and $\mathrm{CBCT}$ images in that axis. 
The information provided by the fiducial markers was also used to analyze the rotations and deformations suffered by the prostate in each treatment fraction. The most relevant rotation was around the lateral axis, which is in agreement with the literature [12] [18] [19] [20] [22] [23]. However, there is controversy about the influence of rotation on treatment margin. Some authors claim that residual rotational can be ignored [19] [22] whereas others state the need to correct with a six-degree-treatment couch or expand the margins to include them [12] [18] [20]. The disagreement could be attributed to the use of different rotation centers. In our study the rotation center is computed in the center of mass of the markers which is very close to the prostate contour centroid. Therefore, the length of lever arm of rotation is close to zero and the effect of the rotation is not relevant. Additionally, some of the cited studies include the seminal vesicles or even the pelvic lymph nodes [12] [18]. This makes the elongation increase and has a relevant impact on prostate coverage.

Our results show that the prostate deformation does not have a great impact on the planning margins, in agreement with other authors [13] [24]. The data that imply a decrease in the distance between the marker and the center of mass were excluded from our statistical analysis, since this reduction should not contribute to the margin. This approach is not taken into account by other authors [7] [25] and may be the reason why margins related to deformations are more relevant in their results. Additionally, it has been shown that the distance between the markers decreases during the treatment course being more pronounced from fraction 13 and in longitudinal axis. Nichol et al. [7] also observed the reduction in prostate volume during treatment by means of MR imaging, one on the day of CT simulation and the second on a randomly assigned fraction; therefore only one patient was chosen by fraction. Using fiducial markers in CBCTs to quantify the volume variation does not require additional image acquisition, removes the uncertainty of prostate surface contouring, and allows us to have a large sample per fraction.

An estimate of the gland movement can be carried out in different ways. One strategy is imaging before and after radiation delivery either by using $2 \mathrm{D}$ images or by CBCT [26] [27] [28], as in this study. There are also systems that allow a real-time observation of the prostate either by acquiring frequent stereoscopic X-ray imaging of implanted fiducials [29] or by locating implanted electromagnetic transponders [30] [31] [32]. Another option is through continuous EPID imaging or the realization of EPID portal images before the irradiation of each treatment field [33] [34]. Real-time observations have shown that prostate movement does not follow a fixed pattern and depends not only on the patient but also on the treatment fraction. However, the general trend is that the prostate moves away from its initial position as time increases [29]. In addition, although the movement can be reversed during the session, the range is approximated reasonable well by measuring the distance between the prostate position at the beginning and at the end the irradiation [33], being the large excursions 
rare [29] [31]. In the present study, the range of motion was measured and a rectangular probability distribution for prostate position was assigned for taking into account that the prostate does not remain in the final position during the entire fraction.

The prostate rotation, deformation, and motion may be related to physiologic processes, such as rectum or bladder fillings. Our study shows that an appropriate preparation protocol minimizes the impact of the changes in rectum or bladder volume on the prostate rotation or deformation. The analysis indicated a weak correlation, whereby patients with a large difference in rectal volume with respect to planning CT are more likely to undergo higher rotations or deformations.

The resulting margins were between 4.4 to $7.3 \mathrm{~mm}$. In general, studies published in the literature calculated margins through systematic or random errors, but considered only a specific component and rarely took into account the mechanical limitations of the treatment unit or the imaging system. Thus, Oehler et al. [28] obtained margins between 5 - $8 \mathrm{~mm}$ combining set-up uncertainty, intrafractional motion uncertainty and contouring error. Mayyas et al. [27] presented planning margins computed for the residual set-up and intrafraction errors of $4-7 \mathrm{~mm}$.

The selection of uniform margins by axis is a limitation of the current study; for example the margin required at the anterior region of the prostate should be lower than the one at the region close to the rectum wall, because this area is subject to greater movements and deformations due to the presence of rectum. The same argument could be applied to the apex and base.

The present work is also limited to geometrical uncertainty in the prostate and does not take into account the effects of including the seminal vesicles. Addition of the seminal vesicles into the treatment volume depends on the stage and its impact on margins depends on the extent to which they are included. Their mobility causes changes of orientation between the prostate and seminal vesicles as it has been reported [35] and higher margins should be considered when including them in the treatment volume.

\section{Ethical Approval}

All procedures performed were in accordance with the ethical standards of the institutional and national research committee and with the 1964 Helsinki declaration and its later amendments.

\section{Informed Consent}

All patients provided signed informed consent to be treated with the radiotherapy treatment scheme used in the study.

\section{Conflicts of Interest}

The authors report no conflicts of interest. 


\section{References}

[1] Zelefsky, M.J., Kollmeier, M., Cox, B., Fidaleo, A., Sperling, D., Pei, X., et al. (2012) Improved Clinical Outcomes with High-Dose Image Guided Radiotherapy Compared with Non-IGRT for the Treatment of Clinically Localized Prostate Cancer. International Journal of Radiation Oncology Biology Physics, 84, 125-129. https://doi.org/10.1016/j.ijrobp.2011.11.047

[2] Zapatero, A., Roch, M., Büchser, D., Castro, P., Fernández-Banda, L., Pozo, G., et al. (2017) Reduced Late Urinary Toxicity with High-Dose Intensity-Modulated Radiotherapy Using Intra-Prostate Fiducial Markers for Localized Prostate Cancer. Clinical Translation Oncology, 19, 1161-1167. https://doi.org/10.1007/s12094-017-1655-9

[3] Van Herk, M., Remeijer, P., Rasch, C. and Lebesque, J.V. (2000) The Probability of Correct Target Dosage: Dose-Population Histograms for Deriving Treatment Margins in Radiotherapy. International Journal of Radiation Oncology Biology Physics, 47, 1121-1135. https://doi.org/10.1016/S0360-3016(00)00518-6

[4] Stroom, J.C., de Boer, H.C., Huizenga, H. and Visser, A.G. (1999) Inclusion of Geometrical Uncertainties in Radiotherapy Treatment Planning by Means of Coverage Probability. International Journal of Radiation Oncology Biology Physics, 43, 905-919. https://doi.org/10.1016/S0360-3016(98)00468-4

[5] O’Neill, A.G., Jain, S., Hounsell, A.R. and O’Sullivan, J.M. (2016) Fiducial Marker Guided Prostate Radiotherapy: A Review. British Journal Radiology, 89, Article ID: 20160296. https://doi.org/10.1259/bjr.20160296

[6] Gustafsson, C., Korhonen, J., Persson, E., Gunnlaugsson, A., Nyholm, T. and Olsson, L.E. (2017) Registration Free Automatic Identification of Gold Fiducial Markers in MRI Target Delineation Images for Prostate Radiotherapy. Medical Physics, 44, 5563-5574. https://doi.org/10.1002/mp.12516

[7] Nichol, A.M., Brock, K.K., Lockwood, G.A., Moseley, D.J., Rosewall, T., Warde, P.R., et al. (2007) A Magnetic Resonance Imaging Study of Prostate Deformation Relative to Implanted Gold Fiducial Markers. International Journal of Radiation Oncology Biology Physics, 67, 48-56. https://doi.org/10.1016/j.ijrobp.2006.08.021

[8] Sharpe, M.B., Moseley, D.J., Purdie, T.G., Islam, M., Siewerdsen, J.H. and Jaffray, D.A. (2006) The Stability of Mechanical Calibration for a kV Cone Beam Computed Tomography System Integrated with Linear Accelerator. Medical Physics, 33, 136-144. https://doi.org/10.1118/1.2143141

[9] Bissonnette, J.P., Moseley, D., White, E., Sharpe, M., Purdie, T. and Jaffray, D.A. (2008) Quality Assurance for the Geometric Accuracy of Cone-Beam CT Guidance in Radiation Therapy. International Journal of Radiation Oncology Biology Physics, 71, 57-61. https://doi.org/10.1016/j.ijrobp.2007.06.086

[10] Ullman, K.L., Ning, H., Susil, R.C., Ayele, A., Jocelyn, L., Havelos, J., et al. (2006) Intra- and Inter-Radiation Therapist Reproducibility of Daily Isocenter Verification Using Prostatic Fiducial Markers. Radiation Oncology, 1, 1-6. https://doi.org/10.1186/1748-717X-1-2

[11] Deegan, T., Owen, R., Holt, T., Roberts, L., Biggs, J., McCarthy, A., et al. (2013) Interobserver Variability of Radiation Therapists Aligning to Fiducial Markers for Prostate Radiation Therapy. Journal of Medical Imagingand Radiation Oncology, 57, 519-523. https://doi.org/10.1111/1754-9485.12055

[12] Shang, Q., Sheplan Olsen, L.J., Stephans, K., Tendulkar, R. and Xia, P. (2013) Prostate Rotation Detected from Implanted Markers Can Affect Dose Coverage and Cannot Be Simply Dismissed. Journal of Applied Clinical Medical Physics, 14, 
177-191. https://doi.org/10.1120/jacmp.v14i3.4262

[13] van der Wielen, G.J., Mutanga, T.F., Incrocci, L., Kirkels, W.J., Vasquez Osorio, E.M., Hoogeman, M.S., et al. (2008) Deformation of Prostate and Seminal Vesicles Relative to Intraprostatic Fiducial Markers. International Journal of Radiation Oncology Biology Physics, 72, 1604-1611. https://doi.org/10.1016/j.ijrobp.2008.07.023

[14] Salembier, C., Villeirs, G., De Bari, B., Hoskin, P., Pieters, B.R., Van Vulpen, M., et al. (2018) ESTRO ACROP Consensus Guideline on CT- and MRI-Based Target Volume Delineation for Primary Radiation Therapy of Localized Prostate Cancer. Radiotherapy and Oncology, 127, 49-61. https://doi.org/10.1016/j.radonc.2018.01.014

[15] Fiorino, C., Reni, M., Bolognesi, A., Cattaneo, G.M. and Calandrino, R. (1998) Intra- and Inter-Observer Variability in Contouring Prostate and Seminal Vesicles: Implications for Conformal Treatment Planning. Radiotherapy and Oncology, 47, 285-292. https://doi.org/10.1016/S0167-8140(98)00021-8

[16] Rasch, C., Barillot, I., Remeijer, P., Touw, A., van Herk, M. and Lebesque, J.V. (1999) Definition of the Prostate in CT and MRI: A Multi-Observer Study. International Journal of Radiation Oncology Biology Physics, 43, 57-66. https://doi.org/10.1016/S0360-3016(98)00351-4

[17] Remeijer, P., Rasch, C., Lebesque, J.V. and van Herk, M. (1999) A General Methodology for Three-Dimensional Analysis of Variation in Target Volume Delineation. Medical Physics, 26, 931-940. https://doi.org/10.1118/1.598485

[18] Amro, H., Hamstra, D.A., Mcshan, D.L., Sandler, H., Vineberg, K., Hadley, S., et al. (2013) Thedosimetric Impact of Prostate Rotations during Electromagnetically Guided External Beam Radiation Therapy. International Journal of Radiation Oncology Biology Physics, 85, 230-236. https://doi.org/10.1016/j.ijrobp.2012.03.020

[19] Mutanga, T.F., de Boer, H.C., van Der Wielen, G.J., Hoogeman, M.S., Incrocci, L. and Heijmen, B.J. (2011) Margin Evaluation in the Presence of Deformation, Rotation, and Translation in Prostate and Entire Seminal Vesicle Irradiation with Daily Marker-Based Setup Corrections. International Journal of Radiation Oncology Biology Physics, 81, 1160-1167. https://doi.org/10.1016/j.ijrobp.2010.09.013

[20] Owen, R., Kron, T., Foroudi, F., Milner, A., Cox, J. and Ducheesne, G. (2011) Interfraction Prostate Rotation Determined from In-Room Computerized Tomography Images. Medical Dosimetry, 36, 188-194.

https://doi.org/10.1016/j.meddos.2010.03.002

[21] Khoo, E.L., Schick, K., Plank, A.W., Poulsen, M., Wong, W.W., Middleton, M., et al. (2012) Prostate Contouring Variation: Can It Be Fixed? International Journal of Radiation Oncology Biology Physics, 82, 1923-1929.

https://doi.org/10.1016/j.ijrobp.2011.02.050

[22] Redpath, A.T., Wright, P. and Muren, L.P. (2008) The Contribution of On-Line Correction for Rotational Organ Motion in Image-Guided Radiotherapy of the Bladder and Prostate. Acta Oncologica, 47, 1367-1372.

https://doi.org/10.1080/02841860802263232

[23] Kupelian, P. and Meyer, J.L. (2011) Image-Guided, Adaptive Radiotherapy of Prostate Cancer: Toward New Standards of Radiotherapy Practice. Frontiers of Radiation Therapy and Oncology, 43, 344-368. https://doi.org/10.1159/000322485

[24] Deurloo, K.E., Steenbakkers, R.J., Zijp, L.J., de Bois, J.A., Nowak, P.J., Rasch, C.R., et al. (2005) Quantification of Shape Variation of Prostate and Seminal Vesicles during External Beam Radiotherapy. International Journal of Radiation Oncology Biology Physics, 61, 228-238. https://doi.org/10.1016/j.ijrobp.2004.09.023 
[25] Mayyas, E., Kim, J., Kumar, S., Liu, C, Wen, N., Movsas, B., et al. (2014) A Novel Approach for Evaluation of Prostate Deformation and Associated Dosimetric Implications in IGRT of the Prostate. Medical Physics, 41, Article ID: 091709. https://doi.org/10.1118/1.4893196

[26] Kron, T., Thomas, J., Fox, C., Thompson, A., Owen, R., Herschtal, A., et al. (2010) Intra-Fraction Prostate Displacement in Radiotherapy Estimated from Pre- and Post-Treatment Imaging of Patients with Implanted Fiducial Markers. Radiotherapy and Oncology, 95, 191-197. https://doi.org/10.1016/j.radonc.2010.01.010

[27] Mayyas, E., Chetty, I.J., Chetvertkov, M., Wen, N., Neicu, T., Nurushev, T., et al. (2013) Evaluation of Multiple Image-Based Modalities for Image-Guided Radiation Therapy (IGRT) of Prostate Carcinoma: A Prospective Study. Medical Physics, 40, Article ID: 041707. https://doi.org/10.1118/1.4794502

[28] Oehler, C., Lang, S., Dimmerling, P., Bolesch, C., Kloeck, S., Tini, A., et al. (2014) PTV Margin Definition in Hypofractionated IGRT of Localized Prostate Cancer Using Cone Beam CT and Orthogonal Image Pairs with Fiducial Markers. Radiation Oncology, 9, 229. https://doi.org/10.1186/s13014-014-0229-z

[29] Xie, Y., Djajaputra, D., King, C.R., Hossain, S., Ma, L. and Xing, L. (2008) Intrafractional Motion of the Prostate during Hypofractionated Radiotherapy. International Journal of Radiation Oncology Biology Physics, 72, 236-246. https://doi.org/10.1016/j.ijrobp.2008.04.051

[30] Li, J.S., Jin, L., Pollack, A., Horwitz, E.M., Buyyounouski, M.K., Price, R.A., et al. (2009) Gains from Real-Time Tracking of Prostate Motion during External Beam Radiation Therapy. International Journal of Radiation Oncology Biology Physics, 75, 1613-1620. https://doi.org/10.1016/j.ijrobp.2009.05.022

[31] Tanyi, J.A., He, T., Summers, P.A., Mburu, R.G., Kato, C.M., Rhodes, S.M., et al. (2010) Assessment of Planning Target Volume Margins for Intensity-Modulated Radiotherapy of the Prostate Gland: Role of Daily inter and Intrafraction Motion. International Journal of Radiation Oncology Biology Physics, 78, 1579-1585. https://doi.org/10.1016/j.ijrobp.2010.02.001

[32] Langen, K.M., Willoughby, T.R., Meeks, S.L., Santhanam, A., Cunningham, A., Levine, L., et al. (2008) Observations on Real-Time Prostate Gland Motion Using Electromagnetic Tracking. International Journal of Radiation Oncology Biology Physics, 71, 1084-1090. https://doi.org/10.1016/j.ijrobp.2007.11.054

[33] Kotte, A.N., Hofmanm, P., Lagendijk, J.J., van Vulpen, M. and van der Heide, U.A. (2007) Intrafraction Motion of the Prostate during External-Beam Radiation Therapy: Analysis of 427 Patients with Implanted Fiducial Markers. International Journal of Radiation Oncology Biology Physics, 69, 419-425.

https://doi.org/10.1016/j.ijrobp.2007.03.029

[34] Månsson Haskå, T., Honoré, H., Muren, L.P., Hoyer, M. and Poulsen, P.R. (2008) Intrafraction Changes of Prostate Position and Geometrical Errors Studied by Continuous Electronic Portal Imaging. Acta Oncologica, 47, 1351-1357.

https://doi.org/10.1080/02841860802256509

[35] de Boer, J., van Herk, M., Pos, F.J., and Sonke, J.J. (2013) Hybrid Registration of Prostate and Seminal Vesicles for Image Guided Radiation Therapy. International Journal of Radiation Oncology Biology Physics, 86, 177-182. https://doi.org/10.1016/j.ijrobp.2012.11.034 